\title{
A Bayesian modeling approach to bi-directional pedestrian flows in carnival events
}

(C) The Author(s) 2017. Published by Higher Education Press. This is an open access article under the CC BY license (http:// creativecommons.org/licenses/by/4.0)

\begin{abstract}
Bi-directional pedestrian flows are common at crosswalks, footpaths, and shopping areas. However, the properties of pedestrian movement may vary in urban areas according to the type of walking facility. In recent years, crowd movements at carnival events have attracted the attention of researchers. In contrast to pedestrian behavior in other walking facilities, pedestrians whose attention is attracted by carnival displays or activities may slow down and even stop walking. The Lunar New Year Market is a traditional carnival event in Hong Kong held annually one week before the Lunar New Year. During the said event, crowd movements can be easily identified, particularly in Victoria Park, where the largest Lunar New Year Market in Hong Kong is hosted. In this study, we conducted a videobased observational survey to collect pedestrian flow and speed data at the Victoria Park Lunar New Year Market on the eve of the Lunar New Year. Using the collected data, an extant mathematical model was calibrated to capture the relationships between the relevant macroscopic quantities, thereby providing insight into pedestrian behavior at the carnival event. Bayesian inference was employed to calibrate the model by using prior data obtained from a previous controlled experiment. Results obtained enhance our understanding of crowd behavior under different conditions at carnival events, thus facilitating the improvement of the safety and efficiency of similar events in the future.
\end{abstract}

Keywords pedestrian flow model, bi-directional interactions, empirical studies, Bayesian inference

Received April 6, 2017; accepted September 19, 2017

S. Q. XIE (凹), S. C. WONG

Department of Civil Engineering, The University of Hong Kong, Hong Kong, China

E-mail: seakay@connect.hku.hk

William H. K. LAM

Department of Civil and Environmental Engineering, The Hong Kong Polytechnic University, Hong Kong, China

\section{Introduction}

Walking is an environmentally friendly mode of transportation. A better understanding of pedestrian activities promotes effective planning of walking facilities, which is particularly important in densely populated Asian cities, such as Hong Kong. Considering that pedestrian movement is usually bi-directional, with properties that vary between types of walking facilities, crowd movement at carnival events has attracted the attention of researchers in recent years. In contrast to pedestrian behavior in many common walking facilities, pedestrians whose attention is attracted by carnival displays or activities may slow down or even stop walking. Therefore, an in-depth understanding of crowd movement at carnival events can help cities organize safe events and anticipate where and when crowd accidents are most likely to occur (Helbing et al., 2007) because extreme crowding in small spaces, such as sports arenas, festivals, and other popular entertainment sites, renders emergency evacuation particularly difficult (Johansson et al., 2012).

Video observations have often been used in recent studies to obtain information on crowd movement at typical mass events. Helbing et al. (2007) analyzed a video recording of a crowd disaster in Mina on January 12, 2006. They noted that the main cause of the disaster was the sudden transition from laminar to turbulent flow rather than the stop-and-go flow. Johansson et al. (2008) further analyzed high-density conditions with reference to the flow of pilgrims onto the Jamarat Bridge in Mina. They concluded that stop-and-go waves occur when flow significantly decreases in a congested situation. Moreover, a further increase in density eventually results in crowd turbulence, which may trigger a crowd disaster.

In addition to video observations, researchers have also developed simulation models of crowd movements. Batty et al. (2003) proposed an agent-based model to simulate the effects of the change in the route of the Notting Hill Carnival held annually in London since 1964. Klüpfel 
(2007) used empirical data from three large events, which include the Hajj pilgrimage to Mina, the 2005 World Youth Day in Cologne, and the egress from a football stadium, to calibrate and verify a crowd dynamics simulation model.

In addition to the investigations of crowd movement at mass gatherings, empirical studies of pedestrian flow in various walking facilities have been conducted in the past few decades. For such investigations, controlled experiments can be designed to cover a full range of model parameters and conditions. For example, Daamen and Hoogendoorn (2003), Hoogendoorn and Daamen (2005), and Kretz et al. (2006a) conducted experiments to measure the pedestrian counter-flow in corridors of various widths. Kretz et al. (2006b) and Helbing et al. (2005) observed self-organized lane formation in a series of controlled experiments on bi-directional pedestrian flow in bottlenecks of various widths. Despite their artificial settings, these studies provided valuable information on pedestrian flow in different site conditions with reference to macroscopic quantities, such as flow, speed, and density. Wong et al. (2010) also performed controlled experiments to measure bi-directional pedestrian flow with an oblique angle. Moreover, data obtained from observational surveys can be used to verify and improve models developed from experimental data. Xie et al. (2013) utilized data collected from video observation at a crosswalk in Hong Kong to investigate the conflict generated by intersecting angles and developed a mathematical model of bi-directional pedestrian flow with oblique angles. Xie and Wong (2015) proposed a generalized model of multi-directional pedestrian flow based on data gathered from another observation of a pedestrian street junction with four-directional pedestrian flow.

Observational surveys not only assist in model development but also provide reliable information on pedestrian flow characteristics over a range of walking facilities and countries. Cheung and Lam (1998) conducted surveys in railway stations in Hong Kong to explore the selection of pedestrians between escalators and stairways. Lam et al. (2002) and Lam et al. (2003) conducted surveys on pedestrian flow at signalized crosswalks and indoor walkways in commercial and shopping areas in Hong Kong. A crowd-dynamics model was developed by Fang et al. (2008) from data gathered while observing passengers exiting a railway station in Wuhan, China, during the Spring Festival. Laxman et al. (2010) analyzed pedestrian-flow characteristics in mixed traffic conditions in a medium-sized city in India. Chen et al. (2010) focused on pedestrian flow in confined passageways and stairways.

Because the walking behavior of pedestrians may differ from site to site, it is well worth investigating the movements of crowds at carnival events to improve the organization and safety of such events (Batty et al., 2003; Brambilla and Cattelani, 2009; Giorgini and Sartori, 2016). Hong Kong's Lunar New Year Market is a traditional carnival event held annually a week before the Lunar New
Year. The movements of crowds can be easily observed in Victoria Park, which traditionally hosts the largest Lunar New Year Market in Hong Kong.

First, we conducted a video-based observational survey of pedestrian flow at the Lunar New Year Market in Victoria Park on Lunar New Year's Eve. Next, a series of data-processing techniques were used to extract information on macroscopic quantities, such as speed, density, flow, and the intersecting angles between streams. Specifically, images were extracted from the original video at $0.2 \mathrm{~s}$ intervals and incorporated into a computer program designed to analyze the positions and displacement of individual pedestrians in the crowd. This dataset enabled us to validate the mathematical model of bidirectional pedestrian movement proposed by Xie et al. (2013) and provided us with further insight into pedestrian behavior at carnival events. With a method similar to that of Xie et al. (2013), we used Bayesian inference to calibrate the model. The information obtained from a controlled experiment by Wong et al. (2010) constituted the prior data.

\section{Data}

\subsection{Site conditions}

As mentioned, the Lunar New Year Market held in Victoria Park is the largest among such markets in Hong Kong. Video recording for this study was conducted on the afternoon of the Lunar New Year's Eve; hence, a high crowd density was observed at the site. This recording helped us to validate the model proposed by Xie et al. (2013) for high crowd densities. The video was taken on the afternoon of January 22, 2012 (Lunar New Year's Eve) from $1 \mathrm{pm}$ to $6 \mathrm{pm}$. A clip approximately one hour long with the greatest crowd density was selected for analysis. As shown in Fig. 1, the crowds occupied a large area. Considering that pedestrians were likely to stop walking when their attention was attracted by the displays, a crossing between two shopping zones was selected as the region of interest (ROI) because more pedestrians were expected to continue walking in this area. The ROI comprised two $3 \mathrm{~m} \times 3 \mathrm{~m}$ subareas in the middle of the crossing, as shown in Fig. 1. The camera was set up on the 8th floor of a building near the site, which is $27 \mathrm{~m}$ above ground level.

\subsection{Video data processing}

To extract the pedestrian trajectories, the video footage was first converted into still pictures at an interval of $0.2 \mathrm{~s}$ per frame. Considering that the ROI is $3 \mathrm{~m} \times 3 \mathrm{~m}$, each pedestrian trajectory comprised 10 positions within the ROI; that is, each pedestrian was tracked for $2 \mathrm{~s}$ as they walked across the ROI. Then, the 10 continuous pictures 


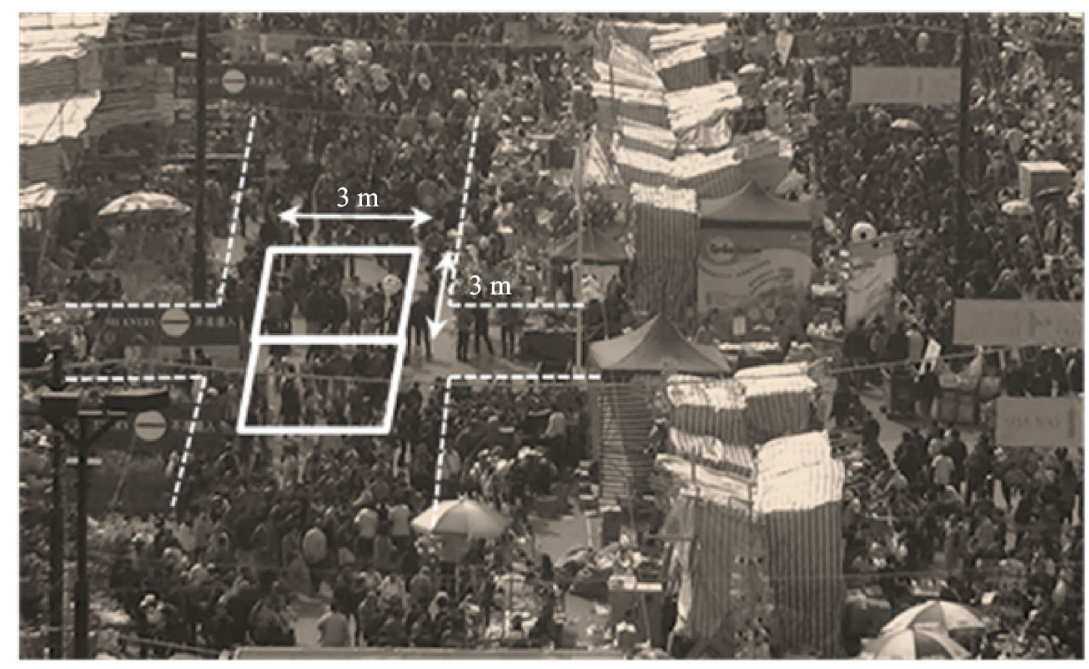

Fig. 1 Lunar New Year Market in Victoria Park

for a $2 \mathrm{~s}$ period were grouped, and the pedestrians within the ROI were manually tracked with a computer program (developed with Visual Basic 6.0). A few student research assistants were appointed to help with the pedestrian tracking.

\subsection{Elimination of "stagnant pedestrians"}

Although the crossing between two zones of shops was selected as the ROI to eliminate most of the pedestrians who were standing still in front of the shops to watch the carnival displays, a few of these "stagnant pedestrians" were still included in the video observation. If the density had been computed with the traditional method, that is, by counting all of the pedestrians in the subarea and dividing the resulting number by the size of the area, then the presence of stagnant pedestrians would have influenced the results. The speed of these pedestrians was nearly zero because they were standing rather than walking. Thus, their presence would have noticeably decreased the average speed calculated for the subarea, thereby resulting in failure to reflect the true movements of pedestrians in that location. Therefore, extracting the moving pedestrian streams from the crowd in the ROI as useful data and eliminating the stagnant pedestrians were particularly necessary for this case study.

The displacement of each pedestrian was measured across every 10 frames to distinguish between pedestrians walking in a certain direction and those standing in a leisurely manner with only slight body movement. A pedestrian is reasonably regarded as "stagnant" if he or she moves only within a very limited area for most of a given period. As reported in the Highway Capacity Manual (National Research Council, 2000), the major and minor axes of the pedestrian body ellipse are 0.60 and $0.50 \mathrm{~m}$, respectively. For convenience, the pedestrian body was defined in this study as a circle with a diameter of $0.50 \mathrm{~m}$, that is, the radius of the pedestrian body was $0.25 \mathrm{~m}$. If a pedestrian had a $95 \%$ chance of moving within only half of the body width surrounding him or her, then he or she was defined as a stagnant pedestrian. In practice, $\sigma$ was computed from the equation $1.96 \sigma=0.25 \mathrm{~m}$ to obtain $\sigma=$ $0.127 \mathrm{~m}$ because $0.25 \mathrm{~m}$ defined the $95 \%$ confidence interval. The position of each pedestrian was traced through the 10 frames, and a pedestrian was considered to be stagnant if the corresponding standard deviation was less than $0.127 \mathrm{~m}$.

The stagnant pedestrians were subsequently eliminated from the computation of average speed and average density. The average speed of a subarea can be calculated by dividing the sum of the speeds of the individual moving pedestrians by the total number of moving pedestrians. However, in this case, the average density could not be ascertained by dividing the number of moving pedestrians by the area of the subarea $(3 \mathrm{~m} \times 3 \mathrm{~m})$ because a certain amount of space was occupied by the stagnant pedestrians. Therefore, the difference between the area of the subarea and the area occupied by the stagnant pedestrians was regarded as the effective area. As previously stated, the radius of the pedestrian body was used again to compute the area occupied by the stagnant pedestrians, which is defined as the sum of their individual body areas. Finally, the average density of the subarea was computed as summarized in Eqs. (1)-(3):

$$
\begin{gathered}
A_{\mathrm{eff}}=A_{\mathrm{s}}-A_{\mathrm{o}}, \\
A_{\mathrm{o}}=m\left(\pi r^{2}\right), \\
\rho_{\mathrm{t}}=\frac{n}{A_{\mathrm{eff}}},
\end{gathered}
$$


where

$A_{\text {eff }}$ is the effective area;

$A_{\mathrm{s}}$ is the area of the subarea, $A_{\mathrm{s}}=3 \mathrm{~m} \times 3 \mathrm{~m}=9 \mathrm{~m}^{2}$;

$A_{\mathrm{o}}$ is the area occupied by stagnant pedestrians;

$m$ is the number of stagnant pedestrians;

$n$ is the number of moving pedestrians;

$r$ is the radius of the pedestrian body $(r=0.25 \mathrm{~m})$; and

$\rho_{\mathrm{t}}$ is the average density (total density).

The average stream density was computed in a similar manner after the directions of the pedestrians were identified.

\subsection{Summary of data}

As shown in Table 1, more than 20000 pedestrian trajectories were traced. The average walking speed was $0.443 \mathrm{~m} / \mathrm{s}$, and the average pedestrian density was 1.16 pedestrian $/ \mathrm{m}^{2}$. The average walking speed was lower than that measured at the crosswalk at Central Station, but the average pedestrian density was higher than the equivalent reading for the crosswalk. The average speed and average density were both lower than those observed in the controlled experiment, which indicates that the pedestrians at the Lunar New Year Market generally walk more slowly as they watch the carnival displays on both sides. Although sufficient space is available for the pedestrians to speed up, they may still opt to walk at this low speed. Nevertheless, validating the proposed model in a high-density range is beneficial because the average density measured in this case was 1.16 pedestrian $/ \mathrm{m}^{2}$ and the maximum density reached a maximum of 5.7 pedestrian $/ \mathrm{m}^{2}$.

\section{Model calibration}

\subsection{Model}

The improved model proposed by Xie et al. (2013) for bidirectional pedestrian movements is as follows:

$$
\begin{aligned}
V_{\mathrm{r}}= & V_{\mathrm{f}} \exp \left(-\theta\left(\rho_{\mathrm{r}}+\rho_{\mathrm{c}}\right)^{2}\right) \exp \left(-\beta\left(1-\frac{V_{\mathrm{r}} \rho_{\mathrm{r}}}{V_{\mathrm{r}} \rho_{\mathrm{r}}+V_{\mathrm{c}} \rho_{\mathrm{c}}}\right)\right. \\
& \left.\times(1-\cos \alpha \varphi)\left(\rho_{\mathrm{r}}+\rho_{\mathrm{c}}\right)\right),
\end{aligned}
$$

$$
\begin{aligned}
V_{\mathrm{c}}= & V_{\mathrm{f}} \exp \left(-\theta\left(\rho_{\mathrm{r}}+\rho_{\mathrm{c}}\right)^{2}\right) \exp \left(-\beta\left(1-\frac{V_{\mathrm{c}} \rho_{\mathrm{c}}}{V_{\mathrm{r}} \rho_{\mathrm{r}}+V_{\mathrm{c}} \rho_{\mathrm{c}}}\right)\right. \\
& \left.\times(1-\cos \alpha \varphi)\left(\rho_{\mathrm{r}}+\rho_{\mathrm{c}}\right)\right),
\end{aligned}
$$

where

$V_{\mathrm{r}}$ is the walking speed of the reference stream;

$V_{\mathrm{c}}$ is the walking speed of the conflicting stream;

$V_{\mathrm{f}}$ is the free-flow walking speed;

$\rho_{\mathrm{r}}$ is the pedestrian density of the reference stream;

$\rho_{\mathrm{c}}$ is the pedestrian density of the conflicting stream;

$\varphi$ is the intersecting angle between the two pedestrian streams;

$\theta, \beta$, and $\alpha$ are coefficients; and

$\frac{V_{\mathrm{r}} \rho_{\mathrm{r}}}{V_{\mathrm{r}} \rho_{\mathrm{r}}+V_{\mathrm{c}} \rho_{\mathrm{c}}}$ is the flow ratio (flow $=$ density $\times$ speed, the momentum term), where $\frac{V_{\mathrm{r}} \rho_{\mathrm{r}}}{V_{\mathrm{r}} \rho_{\mathrm{r}}+V_{\mathrm{c}} \rho_{\mathrm{c}}}=1$ when $\rho_{\mathrm{r}}=0$ and $\rho_{\mathrm{c}}=0$.

\subsection{Calibration results}

Following Xie et al. (2013), we used Bayesian inference to calibrate the model. The prior distributions of the parameters that reflected the interactions between pedestrians $(\theta, \beta$, and $\alpha)$ were those obtained from the controlled experiment. For the free-flow speed, which may depend on the conditions of different walking facilities, an extremely high standard deviation was set to provide sufficient flexibility for the prior distribution.

As shown in Table 2, the calibrated free-flow walking speed was $0.545 \mathrm{~m} / \mathrm{s}(0.95$ confidence interval (CI), 0.536 to 0.553 ), the coefficient of the isotropic effect $\theta$ was 0.050 ( $0.95 \mathrm{CI}, 0.046$ to 0.054$)$, the coefficient of $\beta$ was 0.070 (0.95 CI, 0.062 to 0.079$)$, and the coefficient of $\alpha$ was 1.281 (0.95 CI, 1.215 to 1.345 ).

The values of parameters $\alpha$ and $\beta$ remained stable relative to the parameters calibrated with the data obtained in the controlled experiment and in the observation at the Central Station crosswalk. This outcome indicates that the strength of the conflict induced by the conflicting flow and the intersecting angle of the bi-directional flow remains constant. However, the coefficient of the isotropic effect $\theta$ was 0.05 , which is lower than that obtained in the

Table 1 Summary of data for Victoria Park, the controlled experiment, and the crosswalk at Central Station

\begin{tabular}{lcccccc}
\hline Study & Position & $\begin{array}{c}\text { No. of total } \\
\text { trajectories }\end{array}$ & $\begin{array}{c}\text { Average walking } \\
\text { speed } /\left(\mathrm{m} \cdot \mathrm{s}^{-1}\right)\end{array}$ & $\begin{array}{c}\text { Standard deviation of } \\
\text { speed } /\left(\mathrm{m} \cdot \mathrm{s}^{-1}\right)\end{array}$ & $\begin{array}{c}\text { Average density } \\
/\left(\text { pedestrian } \cdot \mathrm{m}^{-2}\right)\end{array}$ & $\begin{array}{c}\text { Standard deviation of density } \\
/\left(\mathrm{pedestrian} \cdot \mathrm{m}^{-2}\right)\end{array}$ \\
\hline Xie et al. (2013) & $\begin{array}{c}\text { Controlled } \\
\text { experiment }\end{array}$ & 1160 & 0.740 & 0.20 & 2.07 & 0.51 \\
$\begin{array}{c}\text { Crosswalk at } \\
\text { Central Station } \\
\text { This study }\end{array}$ & 6788 & 1.150 & 0.50 & 0.63 & 0.33 \\
\hline
\end{tabular}


Table 2 Calibration results

\begin{tabular}{lccccccc}
\hline Study & Position & CI & $V_{\mathrm{f}} /\left(\mathrm{m} \cdot \mathrm{s}^{-1}\right)$ & $\theta$ & $\beta$ & $\alpha$ & Posterior, $\mathrm{p}$ value \\
\hline Xie et al. & Controlled & Estimate & 1.074 & $\mathbf{0 . 0 6 2}$ & 0.072 & 1.271 & 0.5110 \\
$(2013)$ & experiment & $(95 \%$ CIs $)$ & $(1.065,1.083)$ & $(0.058,0.066)$ & $(0.064,0.080)$ & $(1.208,1.336)$ & - \\
& Crosswalk at & Estimate & 1.326 & $\mathbf{0 . 0 6 5}$ & 0.078 & 0.5028 & 1.214 \\
& Central Station & $(95 \%$ CIs $)$ & $(1.312,1.341)$ & $(0.061,0.069)$ & $(0.070,0.086)$ & $(1.149,1.275)$ & - \\
\multirow{2}{*}{ This study } & Market at Victoria & Estimate & $\mathbf{0 . 5 4 5}$ & $\mathbf{0 . 0 5 0}$ & $\mathbf{0 . 0 7 0}$ & $\mathbf{1 . 2 8 1}$ & 0.5025 \\
& Park & $(95 \% \mathrm{CIs})$ & $(0.536,0.553)$ & $(0.046,0.054)$ & $(0.062,0.079)$ & $(1.215,1.345)$ & - \\
\hline
\end{tabular}

controlled experiment $(0.062)$ and at the crosswalk at Central Station (0.065). Considering that $\theta$ represents the response of the speed to a change in total density, this result indicates that the pedestrian speed at the Lunar New Year Market was less sensitive to changes in total density than that in the controlled experiment and at the crosswalk. It is not surprising that the pedestrians at the market walk slowly to watch the carnival displays despite the presence of sufficient space to speed up. This explanation is also supported by the calibrated free-flow speed of $0.545 \mathrm{~m} / \mathrm{s}$, which is lower than that obtained in the controlled experiment and at the crosswalk. The index posterior $\mathrm{p}$ value, which measures the probability that the replicated data are more extreme than the observed data and is used to evaluate a model's goodness-of-fit, indicates that the fit of the calibrated model is statistically slightly better than that of the original model (values closer to 0.5 indicate a better fit) (Gelman et al., 2014). In summary, this case study reveals that the sensitivity to changes in density may be reduced when the free-flow speed decreases due to other factors, such as attractions on the street, which may influence the speed of pedestrian flow.

\section{Properties of the calibrated model}

Because the model comprises a set of structural equations, computing the speed of the two streams from the known densities and intersecting angles was not straightforward. Therefore, we created a design chart for the intersecting angles $45^{\circ}, 90^{\circ}, 135^{\circ}$, and $180^{\circ}$ to illustrate a few properties of the calibrated model (Fig. 2).

The design chart in Fig. 2, which is constructed as a speed-density diagram of the reference stream, illustrates the general properties of bi-directional pedestrian stream movements; that is, the speed of one stream reduces as its density increases, and the greater the density of the conflicting stream $\left(\rho_{\mathrm{c}}\right)$, the higher the rate of reduction. In contrast to the graphs created for the crosswalk at Central Station, the curves produced from the Lunar New Year Market data begin at a much lower speed. The black solid curve represents the uni-directional pedestrian movements at the intersecting angle $\varphi=0^{\circ}$. The free-flow speed observed at the Lunar New Year Market was $0.55 \mathrm{~m} / \mathrm{s}$, which is approximately half of that observed in the controlled experiment. As previously mentioned, the speed at the market was less sensitive to changes in density; hence, the gradient of the curves is smooth. The curves for the density of the conflicting stream $\left(\rho_{\mathrm{c}}\right)$ at 1,2 , and 3 pedestrian $/ \mathrm{m}^{2}$ are plotted in red, green, and blue, respectively. At $\rho_{\mathrm{r}}=0$, the walking speed of the reference stream $V_{\mathrm{r}}$ drops from 0.51 to $0.23 \mathrm{~m} / \mathrm{s}$ as the density of the conflicting stream $\left(\rho_{\mathrm{c}}\right)$ increases from 1 to 3 pedestrian $/ \mathrm{m}^{2}$. The distances between the starting points of these curves to the free-flow speed $V_{\mathrm{f}}=0.55 \mathrm{~m} / \mathrm{s}$ reflect the conflicting effects induced by the conflicting streams of pedestrians. The intersecting angle between the two streams of pedestrians also influences the conflicting effect. This effect will be even stronger if the conflicting streams of pedestrians are walking in a direction opposite to the reference stream of pedestrians, that is, the intersecting angle $\varphi>90^{\circ}$. As shown in Fig. 2 , the curves for $\varphi=180^{\circ}$ and $135^{\circ}$ lie considerably lower than those of $45^{\circ}$ and $90^{\circ}$ at the same density of the conflicting stream $\left(\rho_{\mathrm{c}}\right)$. Notably, the conflicting effect for the intersecting angle $\varphi=135^{\circ}$ is even larger than that for $180^{\circ}$ because the curve of $\varphi=135^{\circ}$

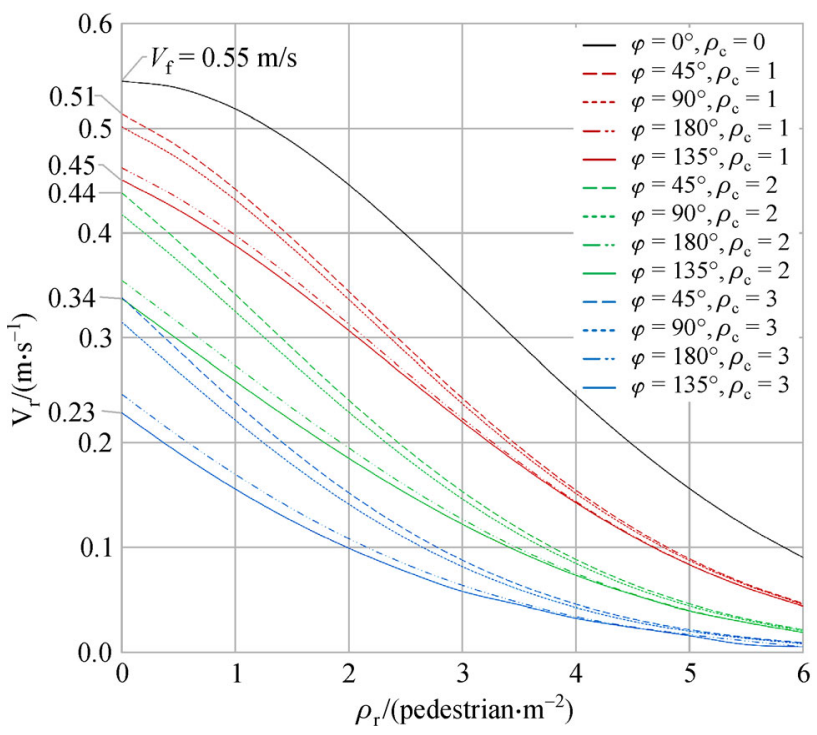

Fig. 2 Design charts for $V_{\mathrm{r}}$ against $\rho_{\mathrm{r}}, \rho_{\mathrm{c}}$ with $\varphi$ of $45^{\circ}, 90^{\circ}, 135^{\circ}$, and $180^{\circ}$ at the Lunar New Year Market in Victoria Park 
always lies at the bottom at each level of $\rho_{\mathrm{c}}$. The walking speed finally drops below $0.1 \mathrm{~m} / \mathrm{s}$ when the density of the reference stream exceeds 6 pedestrian $/ \mathrm{m}^{2}$, thereby indicating that the speed of the pedestrian flow approaches 0 at this density. This case study proves that free-flow speed is dependent on the walking environment because pedestrians can freely speed up or slow down when the crowd density is low, whereas pedestrian movement is substantially confined by the limited space when the density is high.

The flow-total density relationship was plotted for $\rho_{\mathrm{r}}=\rho_{\mathrm{c}}$, as shown in Fig. 3. The optimum total density for the different intersecting angles is approximately 3.0 pedestrian $/ \mathrm{m}^{2}$, with a maximum flow of approximately 0.8 to 1.0 pedestrian $/\left(\mathrm{m} \cdot \mathrm{s}^{-1}\right)$. These values are significantly lower than those observed in the controlled experiment and at the crosswalk because pedestrians at the market walk slowly while watching the carnival displays on both sides.

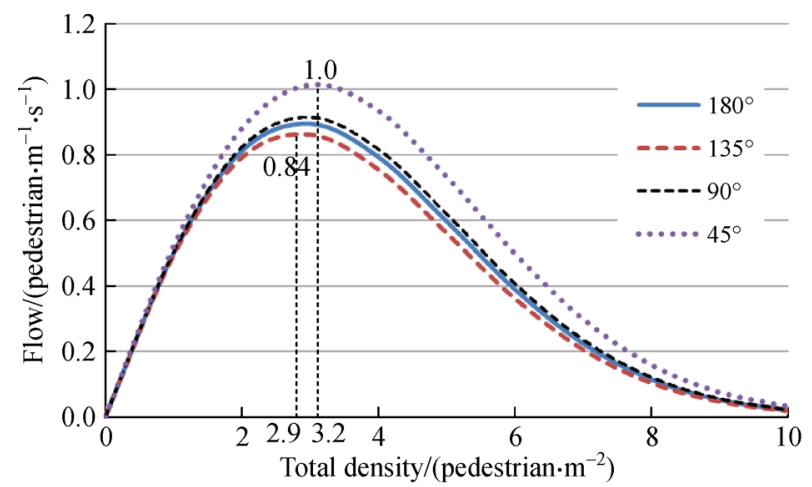

Fig. 3 Flow-total density relationship with different intersecting angles $\left(\rho_{\mathrm{r}}=\rho_{\mathrm{c}}\right)$

\section{Conclusions}

In this study, we conducted a video-based observation of pedestrian movement at a carnival event in Hong Kong and used the data to calibrate the model of bi-directional pedestrian flow proposed by Xie et al. (2013). In contrast to the normal bi-directional flow of pedestrian movement in urban areas, the attention of pedestrians at carnivals may be diverted by various surrounding objects or activities. Therefore, we used the concept of stagnant pedestrians to exclude unmoving and slow-moving pedestrians from the computation of flow and depicted them as static obstructions to the others. The calibrated model is generally consistent with that developed by Xie et al. (2013). However, the free-flow walking speed calibrated for the Lunar New Year Market in Victoria Park was found to be significantly lower than the equivalent speed obtained by Xie et al. (2013) because the former was observed at a carnival event and the latter was obtained at a busy crosswalk in the center of the Hong Kong urban area. The coefficient of the isotropic effect was also low, thereby indicating that the walking speed at the carnival is less sensitive to changes in pedestrian density. This observational study provides us with insightful information on pedestrian movement at carnival events, which can be distinguished from the bi-directional flow of pedestrians in other typical walking facilities in urban areas. Our findings help us understand the behavior of crowds at carnival events, thereby facilitating the improvement of the safety and efficiency of similar events in the future.

Acknowledgements The study described here was supported by a grant from the Research Grants Council of the Hong Kong Special Administrative Region, China (Project No. PolyU 5243/13E). The first and second authors were also respectively supported by the Postdoctoral Fellow Scheme and Francis S. Y. Bong Professorship in Engineering of The University of Hong Kong.

\section{References}

Batty M, DeSyllas J, Duxbury E (2003). The discrete dynamics of smallscale spatial events: Agent-based models of mobility in carnivals and street parades. International Journal of Geographical Information Science, 17(7): 673-697

Brambilla M, Cattelani L (2009). The Distrimobs approach for parallelization of pedestrian mobility computations. Nuovo Cimento Della Societa Italiana Di Fisca C-Colloquia on Physics, 32(2): 105108

Chen X, Ye J, Jian N (2010). Relationships and characteristics of pedestrian traffic flow in confined passageways. Transportation Research Record: Journal of the Transportation Research Board, 2198: 32-40

Cheung C Y, Lam W H K (1998). Pedestrian route choices between escalator and stairway in MTR stations. Journal of Transportation Engineering, 124(3): 277-285

Daamen W, Hoogendoorn S (2003). Experimental research of pedestrian walking behavior. Transportation Research Record, 1828(1): 20-30

Fang Z, Yuan J P, Wang Y C, Lo S M (2008). Survey of pedestrian movement and development of a crowd dynamics model. Fire Safety Journal, 43(6): 459-465

Gelman A, Carlin J B, Stern H S, Rubin D B (2014). Bayesian Data Analysis. 3rd ed. Boca Raton: CRC

Giorgini B, Sartori M (2016). Human mobility world lines on urban topologies. Quality \& Quantity, 50(4): 1817-1831

Helbing D, Buzna L, Johansson A, Werner T (2005). Self-organized pedestrian crowd dynamics: Experiments, simulations, and design solutions. Transportation Science, 39(1): 1-24

Helbing D, Johansson A, Al-Abideen H Z (2007). Dynamics of crowd disasters: An empirical study. Physical Review. E, 75(4): 046109

Hoogendoorn S P, Daamen W (2005). Pedestrian behavior at bottlenecks. Transportation Science, 39(2): 147-159

Johansson A, Batty M, Hayashi K, Al Bar O, Marcozzi D, Memish Z A (2012). Crowd and environmental management during mass gatherings. Lancet Infectious Diseases, 12(2): 150-156

Johansson A, Helbing D, Al-Abideen H Z, Al-Bosta S (2008). From 
crowd dynamics to crowd safety: A video-based analysis. Advances in Complex Systems, 11(4): 497-527

Klüpfel H (2007). The simulation of crowd dynamics at very large events - Calibration, empirical data, and validation. In: Waldau $\mathrm{N}$, Gattermann P, Knoflacher H, Schreckenberg M, eds. Pedestrian and Evacuation Dynamics 2005. Berlin: Springer, 285-296

Kretz T, Grünebohm A, Kaufman M, Mazur F, Schreckenberg M (2006a). Experimental study of pedestrian counterflow in a corridor. Journal of Statistical Mechanics, 2006(10): P10001

Kretz T, Grünebohm A, Schreckenberg M (2006b). Experimental study of pedestrian flow through a bottleneck. Journal of Statistical Mechanics, 2006(10): P10014

Lam W H K, Lee J Y, Cheung C Y (2002). A study of the bi-directional pedestrian flow characteristics at Hong Kong signalized crosswalk facilities. Transportation, 29(2): 169-192

Lam W H K, Lee J Y, Chan K S, Goh P K (2003). A generalised function for modeling bi-directional flow effects on indoor walkways in Hong
Kong. Transportation Research Part A, Policy and Practice, 37(9): 789-810

Laxman K K, Rastogi R, Chandra S (2010). Pedestrian flow characteristics in mixed traffic conditions. Journal of Urban Planning and Development, 136(1): 23-33

National Research Council (2000). Highway Capacity Manual. Washington: National Research Council

Wong S C, Leung W L, Chan S H, Lam W H K, Yung N H C, Liu C Y, Zhang P (2010). Bidirectional pedestrian stream model with oblique intersecting angle. Journal of Transportation Engineering, 136(3): 234-242

Xie S, Wong S C, Lam W H K, Chen A (2013). Development of a bidirectional pedestrian stream model with an oblique intersecting angle. Journal of Transportation Engineering, 139(7): 678-685

Xie S, Wong S C (2015). A bayesian inference approach to the development of a multidirectional pedestrian stream model. Transportmetrica A: Transportation Science, 11(1): 61-73 